\title{
HIST ORY
}

OF A

\section{REMARKABLE CASE}

or

\section{VARICOSE ANEURISM,}

WITH OBSERVATIONS.

\section{BY JOHN GEORGE PERRY,}

SURGEON TO THE FOUNDLING HOSPITAI, AND TO THE ST. MARYLEBONE INFIRMARX.

\section{READ NOV. $24 T H, 1835$.}

The subject of varicose aneurism has been lately so fully and ably treated by M. Breschet*, and its pathology has received from him so much patient and acute investigation, that the present state of our knowledge furnishes little which can be added to his history of the disease. A fact, however, has lately come under my observation, which is not adverted to as even possible, either by M. Breschet, or any preceding writer that $I$ am acquainted with, and which, from its practical importance, seems to me of sufficient moment to be made the subject of a separate communication to the Society.

* Mem. de la Société Royale de Médecine, T. III.p. 198 et seq. 
All writers on this disease, from Dr. Wm. Hunter, who first described it, to the celebrated surgeon I have already alluded to, seem to have regarded a wound of the vessels by some penetrating instrument as a necessary preliminary to the establishment of that permanent channel of communication, through which the intervascular transfusion ever afterwards takes place; indeed, the previous wound is in all instances assumed, as a necessary condition, in the definition of the disease. "Il faut," says M. Breschet, " que la veine accompagnant l'artère, et qui est accolée à ses parois soit traversée de part en part, avant que l'instrument atteigne l'artère." Such a definition was undoubtedly quite borne out by all the cases hitherto recorded; and the great indisposition manifested by blood-vessels, and especially veins, to partake of the ulcerative process, even when it may have involved all the surrounding tissues, rendered it very improbable that an opening would take place in the coats of one of the last mentioned vessels from any of the ordinary causes of absorption. Aneurisms of large arteries have often been seen to cause, by their pressure, absorption of all the solid structures around, without their accompanying veins becoming, in any degree, involved in the general havoc. The case which is about to be related to the society, however, will suffice to prove that such laws, though of general, are not of universal application.

Jonathan Allum, ætat. 47, in the early part of his life was a private of dragoons, and served ir the Peninsular war. He was never a very strong man, 
and was discharged from the army in the year 1819, on account of general ill-health. He had no recollection of any accident having occurred to him except one, a few years before he left the army, when he and his horse were knocked down by the fall of a heavy piece of timber. His left knee was much hurt at the time, and had never since been as strong as the other, and sometimes had been so painful as to lead him to apply for surgical assistance at the infirmary.

In the year 1831, being at that time employed in drawing a truck, he suffered for several months from pain in the foot, at its inner side, and matter formed, and was discharged from that part. Sometime in the course of the same year he first perceived a small swelling a little below his left knee, but it gave him no pain and he disregarded it. The swelling increased very slowly in size and troubled him very little, that he could recollect, for two or three years, except that occasionally, when drawing his truck, he was seized with pain in it to such a degree as to be obliged to stop for a few minutes from his work.

About two years after the first discovery of the tumour, his wife accidentally perceived a palpitation (to use his own phrase) in the middle of the left thigh, which alarmed her very much, as she connected it in her mind with a palpitation he was then subject to about his heart, and which was sometimes so. violent, as to prevent him from doing any work. The patient himself, however, suffering no inconvenience from the condition of the thigh, paid no attention to it. The palpitation in the thigh went on increasing. vol. $\mathrm{xx}$. 
as did also the swelling in the leg, nothing being done for their relief until Feb. 6th, 1834, when he applied for admission into the infirmary, chiefly on account of pain which had come on again in the inner side of his foot and great toe, and which he considered his most severe complaint.

Upon examination soon after his admission, an aneurismal tumour of considerable size was discovered at the upper part and inner side of the calf of the left leg, apparently occupying the lower end of the popliteal, or the commencement of the posterior tibial artery. The contents of the sac were so entirely fluid, that it could be almost emptied by pressure with the hand, maintained for a few minutes. Pulsation could be felt distinctly in the anterior tibial artery as it passed over the tarsus, and obscurely in the posterior tibial, behind the internal malleolus.

As the patient lay on his bed with the limb placed on its outer side, a very remarkable pulsation could be distinctly seen along the course of the femoral artery and vein throughout the greater part of the upper two-thirds of the thigh, beginning about two inches below the crural arch, and terminating at the spot where the femoral vessels become included in the tendinous sheath formed by the triceps muscle. When the hand was laid upon any part of this region a very peculiar thrilling was perceived, occupying a space of at least two inches on either side of the vessels, but varying in intensity and force according to the distance of the part examined from them. I know not how to describe the sensation communicated 
to the hand better than by borrowing a simile from Laennec *, who, in describing the sounds attendant on a contracted state of the left auriculo-ventricular opening, says, " on sent quelquefois à la main un frémissement analogue à celui qui accompagne le murmure de satisfaction que font entendre les chats, lorsqu'on leur passe la main sur le dos." This purring (to adopt his phrase) was quite distinguishable from the pulsation of the artery; for while the latter was lost in the intervals corresponding to the diastole of the heart, the former continued to be felt without intermission, deriving only renewed intensity from the repetition of the heart's action.

Various suggestions were offered by the many experienced surgeons who, in addition to my colleagues, did me the favour to examine the case with me, with the view of explaining the phenomenon $I$ have endeavoured to describe. Some supposed it to be occasioned by an unusual condition of the lining of the artery; others attributed it to the dilated state of the vessel; whilst another gentleman ascribed it to the escape of blood from the artery into the sheath of the vessels, which he supposed might have there formed an elongated aneurismal sac. Notwithstanding the difference of opinion which prevailed as to the nature of the affection, these gentlemen all coincided with me in opinion, that the diseased state of the vessel rendered it inexpedient to place a ligature upon it, until the increase of the tumour in the ham should

- Traité de l'auscultation médiate, Tom. II., p. 215, 1st edit. 
have brought the patient's life into immediate danger. Neither did the ligature of the external iliac artery promise a more successful event, since its pulsation could be felt and heard over so wide a space, that it also was conceived to be greatly dilated, if not decidedly aneurismal.

In one of the many examinations I made of the limb during the patient's residence in the infirmary, I chanced to discover, that by pressing the point of my finger upon a part of the artery immediately before it entered the sheath of the triceps, I could entirely stop the purring, without interrupting the circulation through the vessel. This discovery having naturally suggested the idea of the existence of a communication at this part between the artery and vein, the patient was very closely interrogated, to ascertain whether he had ever suffered a wound in that situation. He, however, constantly denied that he had had any such accident: neither was any scar visible on the skin.

It having been decided that no operation should, in the present circumstances, be attempted for the relief of the disease, the general treatment during the patient's sojourn in the infirmary, was confined to such means as were best calculated to diminish the force of the circulation, while rest in the recumbent posture was most strictly enjoined. For some time after his admission, pressure, by means of a bandage extending from the foot nearly to the groin, was attempted to be maintained, but it was eventually discontinued on account of the uneasiness it occasioned. 
For this was substituted the more circumscribed pressure of a spring, resembling a truss, having at its extremity a small pad, intended to imitate as nearly as possible the pressure of the point of the finger, which was so effectual in restraining the purring. It was hoped by the employment of this instrument to effect obliteration of the presumed communication between the vessels, and thus to induce such a condition of the parts, as would justify the having recourse to the ligature of the femoral artery, for the cure of the aneurism in the ham;-whilst it was thought possible that the retardation of the circulation through the artery might even afford occasion for the natural cure taking place in the sac.

After a residence of nine months in the infirmary, as no change was observed in the condition of the diseased parts, the patient was permitted, at his urgent request, to return to his home, promising that he would there continue the employment of the means just described, and he was accordingly discharged on the 24.th of November, 1834, from which time until September last he was regularly visited by the assistantsurgeons of the infirmary. During this interval I occasionally saw him and could perceive no change in the size or other characters of the popliteal tumour, but the purring in the thigh was obviously diminishing in force and extent. On the $22 d$ of July I examined the limb with great attention, with a view to this point :-the purring was then not perceptible for more than an inch on either side of the sheath of the vessels, neither could it be seen, as before; but 
required for its discovery that the hand should be applied to the surface. The superficial veins of the leg had become remarkably enlarged.

I may be permitted here to anticipate the subsequent details of the case, so far as to say that these changes were sufficiently explained on dissection by the obliteration which was found to have been brought about in the vein, probably by the long continued pressure, and which, by diminishing the bulk of the circulating contents of the vessel, rendered that remarkable bruissement less obvious than before. The obliteration of the femoral vein also accounted for the enlargement of the superficial veins.

At this time the tumour in the ham was little, if at all, increased in size; but on the evening of the 9th of September, the patient applied to be re-admitted into the infirmary, on account of its great and sudden enlargement, which he described to have taken place a few days before, and to have been preceded by much pain and throbbing.

At my visit to the infirmary on the following morning, I found the tumour in the ham increased to three or four times its former size, but still retaining its original form. The skin covering the tumour was extremely tense, and presented, at its most prominent part, a livid discoloration, which seemed to threaten almost immediate sloughing. Great pain was felt in the tumour and all down the leg, and the superficial veins were much enlarged and distended with blood. It was now evident that the patient's life would be very shortly lost by hæmorrhage, unless something were 
done without delay for his relief; and though the probable existence of disease in the whole artery rendered the prospect of relief, from any operation, very questionable, it was obviously incumbent upon me to give the patient the advantage of the slender chance presented by the ligature of the vessel. The operation was accordingly performed on the following day, in the usual situation, at the inner margin of the sartorius muscle. Upon dividing the integuments and sheath of the vessels, the artery was found to be enlarged nearly to the size of the abdominal aorta, and its coats so remarkably thin, that it presented more of the appearance of a vein, than of an artery. Much difficulty being experienced in carrying the point of the needie round the artery, apparently from adhesion at its back part, and its extreme tenuity rendering it very hazardous to employ any degree of force, it was deemed advisable to enlarge the opening in the sheath, and pass the ligature about half an inch higher up, which was effected without any difficulty. So attenuated and fragile were the coats of the vessel, that they actually gave way under the slight pressure made by the fingers of a gentleman, who held it with the view of assisting in the passage of the needle under it, and blood issued in a minute jet from its anterior surface. This accident made it necessary to observe the greatest caution in tightening the ligature, as it seemed not improbable that if drawn with ordinary force, it would completely divide the artery. The noose was therefore only drawn to a sufficient degree to stop the pulsation in the sac 
and lower portion of the artery, and as the bleeding from the small laceration before described immediately ceased, it was deemed that enough had been accomplished to stop the circulation through the vessel, and the wound having been closed by straps of adhesive plaster, the patient was carried back to bed. The vein was not seen during the operation, being completely concealed by the enormous dilatation of the artery.

It being my object to dwell only on the points of peculiarity presented by this case, I forbear to trespass on the time of the Society by detailing its daily progress, or describing characters which it presented in common with other cases of popliteal aneurism. It will suffice to state that about four hours after the operation feeble pulsation was perceptible in the artery below the ligature;-that on the following day, (Sept. 12th,) it was somewhat stronger, and that the feeling of purring had returned, being, however, limited to the point where the artery was about to enter the sheath of the triceps;-that during the 13th, 14th, and 15th, these phenomena, though somewhat fluctuating, were, upon the whole, increasing in force and extent, and that at the same time feeble pulsation could be heard, though not felt, in the popliteal tumour. 'The limb, shortly after the operation was warmer than its fellow, and continued throughout to maintain its superior temperature, and the pain which, prior to the operation, had been felt in the tumour and down the leg, was almost entirely relieved.

In the night of the 15th, hæmorrhage suddenly 
took place from the wound to a very large amount, inducing a state of syncope, from which the patient was with difficulty roused. The bleeding was on this occasion restrained by pressure on the wound, but recurred on the following day, when the patient sank exhausted, on the sixth day after the operation.

Dissection.-The lungs were adherent in many parts to the costal pleura and presented several old cavities which were cicatrised. The pericardium was universally adherent to the surface of the heart. The right auricle was large-right ventricle proportionally small-the left ventricle dilated and hypertrophied. The tricuspid and mitral valves were healthy, as well as those of the pulmonary artery and aorta. In the coats of the aorta, at its commencement, at the arch, and near its bifurcation, was deposited much of the atheromatous matter usually found in the large arteries of aneurismal subjects. A single pointed spiculum of bone presented itself among the atheromatous matter at the arch. The aorta was much dilated about the origin of the arteria innominata, which vessel was unusually large. The abdominal aorta was rather thin in its coats.

The external iliac arteries, especially the left, were extremely tortuous, being reflected upon themselves in a singular manner, during their course towards the crural arch : a condition which had, no doubt, given rise to the impression of the artery being extremely dilated, since it conveyed to the hand simultaneously the combined pulsations of the folded portions.

The coats of the femoral artery, throughout its 
whole course; were scarcely, if at all, thicker than those of a vein, the attenuation having, as careful dissection afterwards proved, taken place equally in all its coats. Immediately below the origin of the profunda the vessel was greatly dilated, having the appearance of an aneurismal sac. Its coats were here softened and much attenuated, and presented an aperture anteriorly, large enough to admit the point of the ring-finger, from which the fatal hæmorrhage had taken place. The ligature had been placed at a very short distance below this part of the vessel. The wound was full of coagulum, which had also made its way into the sartorius and surrounding muscles, and by its pressure had probably restrained the hæmorrhage so as to prevent the patient from bleeding to death at its first access.

At the spot in the thigh where the communication had been presumed to exist between the artery and vein, there was an aneurismal sac, about as large as half a walnut, firmly ossified within, which, by the pressure it had exerted upon the vein, had caused absorption of its coats, so as to form a circular opening of about two lines in diameter, into which the aneurism had burst, - thus inducing a free and persistent communication between the vessels. Just below the aperture the vein was obliterated at a single point, below which it was again pervious. In all the rest of its course up the thigh it was diminished in size and thickened.

The changes above described, which took place in the limb between the time of the patient quitting the 
Infirmary and his re-admission, leave no room to doubt that the obliteration of the vein was accomplished in that interval, by the use of the spring, which effectually compressed the vessel under the bony walls of the aneurism.

At the lower part of the popliteal artery, exactly at the point of division into the anterior and posterior tibial trunks, the vessel was dilated into a very large aneurismal sac, which contained a small quantity of laminated fibrine; but the greater part of its contents consisted of loose coagula and serum. The tibia formed a part of the walls of the sac, and was partly absorbed, so as to present a scabrous surface. The posterior tibial artery opened from the sac, as the popliteal had opened into it, by a circular and even orifice. The anterior tibial could not be examined, as it was sacrificed in separating the tumour from the tibia.

The preparation of the diseased parts is preserved by my friend Mr. Stanley in the Museum of St. Bartholomew's Hospital.

In reflecting upon the circumstances of the above case, we are at first sight inclined to feel less surprise that such a complication of aneurism should sometimes occur, than that it should occur so rarely. The proximity of the venæ comites to most of the great arterial trunks, naturally points them out as the parts most likely to be involved in the destruction occasioned by the growth of aneurisms; and nothing would seem more easy, than that such a dilatation of an artery should finally burst into the accompanying vein. As far, however, as I have been able to ascer- 
tain, no instance has been hitherto recorded of such an accident having taken place in any of those arteries with whose diseases the surgeon is especially concerned-namely, in those of the extremities. Experience shows that pressure on a vein is far more likely to cause obliteration of the vessel, by the effusion of lymph from its internal surface, than either ulceration or sloughing; nor are there wanting numerous incidental proofs of the indisposition of these vessels to partake of morbid changes of the latter kind, in the history of other diseases and accidents. In support of this remark, I may allude to two cases of a different nature, which occurred under my observation, and which tend to show how little obnoxious the veins are to these destructive changes.

The first was that of a woman affected with sloughing phagedæna, in St. Bartholomew's Hospital, in the year 1823, when I held the office of house-surgeon to that charity. After having destroyed the labia, and almost insulated the lower part of the rectum, the disease extended its ravages upwards into the groin, and having laid bare, without opening, the femoral vein, at length destroyed the patient by ulceration of its accompanying artery.

The other instance to which I may briefly advert, was that of a young woman, lately brought into the St. Marylebone Infirmary, in consequence of having been run over by a cabriolet, which had severely lacerated the inner side of the leg. Sloughing ensued of the fascia and muscles, almost down to the bone, and the internal saphæna vein was exposed in full three inches of its length; yet, notwithstanding its almost 
entire insulation, it remained unaffected, and was not even obliterated during the granulation and cicatrization of the wound.

The occurrence of so rare a complication in the case just related, is probably due to the circumstance of the aneurism having arisen at a part, where (beyond all other situations in the extremities) the vessels are enclosed in a sheath, so dense and unyielding, as to preclude the possibility of the vein undergoing displacement. Thus, it appears to me, may be satisfactorily reconciled the apparent anomaly of the vein having, in this instance, become obnoxious to the ordinary results of great and long-continued pressure.

I cannot conclude these remarks, without pointing out the confirmation furnished by the case of Jonathan Allum, of the opinion of Mons. Breschet*, that the thinning of the arterial_coats, and the dilated state of the vessel above the opening of communication, are consequences of the long prevalence of the varicose aneurism. I am not aware of any facts proving the constant existence of these changes in arteries predisposed to true aneurism, while dissection has almost invariably shown their presence in cases of the varicose form of the disease. The concomitant thickening of the coats of the vein in the case here narrated strongly corroborates the opinion, that these alterations are in some manner due to the reciprocal interchange of the contents of the vessels, which is proved to take place.

$$
\text { - Op. cit. p. } 206 .
$$

6, Great James Street, Bedford Row, Nov, 23, 1835. 